\title{
75 años de Fenomenología de la percepción: un homenaje local, o la localidad como tarea filosófica Introducción
}

Fenomenología de la percepción es tal vez uno de los textos más contemporáneos de la filosofía francesa contemporánea. No sólo por la radicalidad de sus análisis, hace tres cuartos de siglo tan potentes como ahora, sino por la actualidad que es posible relevar en ellos: el estudio profundo, detallado, sensible, desprejuiciado, de la experiencia corpórea. Tal vez uno de los puntos más novedosos de la filosofía de los últimos cuarenta años, y uno de los cambios socio-políticos más destacados de nuestras sociedades contemporáneas.

Estos análisis, escritos en la pesadumbre de una París ocupada, nos hablan hoy, en el primer tercio del siglo XXI, con la misma intensidad que en la primera mitad del XX. Publicada en 1945, Fenomenología de la percepción inaugura la posguerra con muchas preguntas. Preguntas que se expanden a toda una generación, situada en enclaves existenciales diferentes pero convocada por este pensamiento nuevo, desde la África de Fanon a la vanguardia mexicana, pasando por la Facultad de Filosofía y Letras de la Universidad de Buenos Aires. En un aniversario marcado por las celebraciones a nivel mundial, el presente dossier busca relevar los trabajos de algunos de los especialistas locales en el pensamiento de Merleau-Ponty, y ofrecer de este modo materiales para comprender la profundidad de un pensamiento que no sólo eleva la categoría de situación existencial al rango filosófico, a concepto o existenciario, sino que nos obliga también a pensarla desde una dimensión concreta y corpórea, ligada a un suelo y a una historia particulares. Como bien lo expresa el mexicano Emilio Uranga en 1948, año de la única visita de Merleau-Ponty a América Latina:

En definitiva, lo que decide el valor del existencialismo es su capacidad de dar base a una descripción sistemática de la existencia humana, pero no de la existencia humana en abstracto, sino de una existencia humana situada, en situación, de una existencia humana encuadrada en un hábitat geográfico determinado, en un cuadro social y cultural también determinado y con un legado histórico preciso (Uranga, 1948: 240). ${ }^{1}$ 
En México, en Argentina, en Brasil, la historia de la incorporación de este pensamiento es tan disímil como la de Francia, Italia, o Alemania. Ninguna de estas recepciones fue igual, tuvo el mismo sentido o dialogó con las mismas tradiciones.

Pensemos sólo en el caso de América Latina. El citado Uranga, traductor en 1957 de la Fenomenología de la percepción, una de las figuras centrales en la incorporación del pensamiento merleau-pontyano en México, fue formado por el el gran traductor español de corazón mexicano José Gaos, de un marcado gusto por la filosofía heideggeriana. Uranga, tomando distancia de su maestro y de cierto eurocentrismo académico, forma parte de esa generación que conformará el destacado grupo Hiperión (en cuyas filas se encontraban, entre otros, Luis Villoro), movimiento de vanguardia estético-política centrada en la comprensión (autognosis) de la cultura mexicana. Es allí donde el pensamiento merleau-pontyano, un poco subestimado por los fenomenólogos de academia, encuentra toda la potencia de un pensamiento vivo ligado fuertemente a una filosofía situada, preocupada por su cultura, desde una perspectiva también política y estética.

Unos años más tarde, en Brasil, una joven Marilena Chauí escribe su tesis de maestría, un lustro después de la muerte de Merleau-Ponty y bajo la guía del gran filósofo brasileño Bento Prado Jr. Ese primer acercamiento al pensamiento merleau-pontyano, del que nunca se alejará, se concentra en su pensamiento político. Más precisamente, esta primera tesis de la joven Chauí sobre Merleau-Ponty tiene por objeto el problema y la crítica al humanismo. Lejos de las vanguardias estéticas pero también del pensamiento fenomenológico duro, la mirada de Chauí, rigurosamente filosófica pero fuera de la tradición fenomenológica y alemana, colocó el pensamiento del filósofo francés en el campo brasileño en otra red de tradiciones, desde Espinoza a Deleuze pasando por Marx. Y también en otra red de lectores, pues la figura de Marilena Chaú, con una vida pública muy activa en la cultura brasileña, excede ampliamente la academia. Luego, a partir del trabajo de estudiantes que ella misma fue formando, puede rastrearse también otra lectura en Brasil, enmarcada en la fenomenología y signada por una dura línea que va de Husserl a Merleau-Ponty (aunque no tan ligada a la escuela fenomenológica de manera horizontal, como será el caso en Argentina) pasando por algunos pensadores racionalistas o antirracionalistas clásicos, de Descartes a Nietzsche. Este es el caso por ejemplo de Carlos Alberto Ribeiro de Moura, quien traducirá Fenomenología de la percepción al portugués de Brasil en 2006.

En Argentina, un también joven Roberto Walton escribe hacia 1966, en el mismo momento que su colega brasileña, una tesis de licenciatura bajo la dirección de su maestro y uno de los grandes introductores de la fenomenología en Argentina, Eugenio Pucciarelli. Centrada en el problema del lenguaje, la lectura waltoniana de Merleau-Ponty influirá, a partir de entonces, a innumerables generaciones de filósofos y filósofas argentinos, en el marco de una estricta lectura técnico-filosófica del pensamiento fenomenológico, pero extremadamente abarcativa de la escuela, sin distinción jerárquica ni oposiciones entre los autores. En la formación waltoniana, lo que prima es la escuela: de Ricoeur a Heidegger, de Fink a Patocka, de Marion a Barbaras, de Scheler a Levinas, todos los autores conviven y dialogan en una perspectiva de conjunto guiada por una impresionante erudición de la obra del fundador de la escuela, Edmund Husserl. Este trazo profundamente constructivo de la lectura de Roberto Walton, sumado a su enorme rigor técnico-filosófico, es uno de los más distintivos y, desde mi punto de vista, más productivos aspectos de su enseñanza y escritos. Por otra parte, en su lectura de la fenomenología, y por tanto también del pensamiento de Merleau-Ponty, prima un diálogo en relación con cierta tradición "idealista" que, partiendo de Descartes, y siguiendo por el empirismo inglés, se continúa en la línea alemana que va de la filosofía trascendental al idealismo absoluto, sin necesidad de pasar por Marx. 
Este último acercamiento sucederá, sin embargo, en el campo intelectual porteño, pero por otras vías: a través una serie de pensadores y ensayistas que retomarán al filósofo ya no en su carácter de fenomenólogo sino de pensador existencialista, el más destacado de los cuales es el caso de León Rozitchner, alumno de Merleau-Ponty en la Sorbonne en los años 1950 y traductor temprano de Humanismo y terror (Buenos Aires, Leviatán, 1956) y Las aventuras de la dialéctica (Buenos. Aires, La pléyade, 1974). Rozitchner incorpora algunos conceptos fundamentales del pensamiento merleaupontyano y los lleva al campo de una interesante y original reflexión filosófica ligada a la cultura, al marxismo, al psicoanálisis o al judaísmo. Junto con otros autores, entre los que se puede mencionar a un Oscar Masotta, comparte la característica de haber desarrollado su pensamiento fuera del estricto marco de la enseñanza académica de la Facultad de Filosofía y Letras de la Universidad de Buenos Aires, pero de haber sido también formados por ella.

Pues bien, los textos aquí reunidos recogen y redefinen, en cierto modo, estas primeras improntas a partir de seis escritos y un informe bibliográfico sobre la actualidad de los estudios merleau-pontyanos en Argentina.

El escrito que inaugura el dossier, del propio Roberto Walton, nos introduce en una de las filosofías más originales e injustamente olvidada de nuestra historia intelectual, la primera en retomar e interpretar el pensamiento de Merleau-Ponty en la Facultad de Filosofía y Letras de la Universidad de Buenos Aires. Se trata de una primera incorporación del pensamiento merleau-pontyano ligada al proyecto de una estética filosófica: el proyecto de Luis Guerrero. En "Nota sobre la introducción de la fenomenología de Merleau-Ponty en la Argentina. La importancia de la Estética de Guerrero", Walton retoma las primeras recepciones del fenomenólogo francés en el campo intelectual local y se detiene en la Estética de Guerrero, y en el lugar que el pensamiento merleau-pontyano del lenguaje ocupa en ella. Del mismo modo, releva la importancia de la experiencia no-objetiva y de la experiencia común e histórica en esta obra, deudoras también del pensador francés.

Ya desde el punto de vista de la obra que nos convoca, Fenomenología de la percepción, el texto de Esteban García, "El cuerpo sexuado, el sombrero con pluma y el automóvil. Phénoménologie de la perception leída desde una perspectiva feminista y performativa", desarrolla las posibilidades de la noción de "cuerpo sexuado" tal como allí aparece. García pone en discusión esta perspectiva con la de otros estudios contemporáneos sobre el cuerpo, el género y la sexualidad, más específicamente con la teoría performativa del género desarrollada por Judith Butler, para mostrar los límites de las críticas dirigidas a la teoría merleau-pontyana del cuerpo y señalar, en cambio, su confluencia con la posición del fenomenólogo en vistas a una teoría no esencialista del cuerpo.

Desde una perspectiva histórico-metafísica, mi artículo "Del movimiento de los entes naturales a la dinámica transpersonal del viviente humano: ¿quién es el sujeto de percepción?" retoma el problema del cuerpo en su relación con el movimiento, el tiempo y la vida personal. A partir de un recorrido por el problema de la relación entre cuerpo y movimiento, concluye sobre las diversas dimensiones temporales que el movimiento corporal presenta en Fenomenología de la percepción, y propone, a partir de la potencia crítica que estos diversos niveles aportan, la búsqueda de una nueva noción de vida personal.

El trabajo de Ariela Battán-Horestein, por su parte, se sitúa en una reflexión de carácter más bien epistemológico sobre los alcances, los límites y, sobre todo, los diálogos posibles entre la fenomenología merleau-pontyana del cuerpo, tal como es entendida en Fenomenología de la percepción, y los desarrollos de las llamadas ciencias cognitivas durante los últimos años. A través de un paciente trabajo de traducción, 
Battán-Horenstein intenta poner de relieve malentendidos, insuficiencias y prejuicios reinantes en este diálogo, y aboga por señalar las ganancias de una verdadera escucha entre ambas perspectivas.

Graciela Ralón aborda otro aspecto abierto por Fenomenología de la percepción que conduce también a un diálogo contemporáneo con las ciencias, pero esta vez humanas o del espíritu, como quiera uno situarse en términos de tradiciones. En "El acontecimiento como enigma y huella" se trata del problema del acontecimiento, de sus abordajes y de sus posibilidades. En una interesante lectura, Ralón parte del problema de la historia en la obra del ' 45 hacia la cuestión del acontecimiento histórico, de sus características propias y, desde allí, reflexiona sobre la cuestión del acontecimiento tal como es abordada en las discusiones actuales, principalmente ligadas al ámbito de la fenomenología, y señala los aportes que la noción merleau-pontyana introduce en este debate.

El trabajo de Leonardo Eiff retoma algo de aquella otra tradición que, desde el pensamiento político y social, recibió la obra de Merleau-Ponty y la puso en discusión con las ciencias sociales. En "Trazos de lo político a partir de Fenomenología de la percepción", Eiff desarrolla una perspectiva original respecto del diálogo tradicional del fenomenólogo con la filosofía y el pensamiento político: dejando en suspenso los clásicos acercamientos a través del marxismo y la dialéctica de la historia, inscribe las cuestiones políticas arraigadas en Fenomenología de la percepción y las sitúa en dos ejes problemáticos diferentes, ligados a tradiciones del pensamiento político diversas: por un lado, la tradición que articula la tríada libertad-poder-espacio; por otra, la de institución-autoridad-tiempo. En ambas, el autor pone en discusión la figura del cuerpo político esbozada por Merleau-Ponty y señala su potencia crítica al interior de estas tradiciones conceptuales.

Finalmente, el informe bibliográfico realizado por Claudio Cormick y Cintia Mariscal cierran este panorama general de la producción de estudios merleau-pontyanos de autores argentinos que se inscriben, de forma pensada o impensada, en la tradición abierta por este pensamiento.

Anclaje histórico; apertura de la filosofía hacia la no-filosofía tal como la entendía el propio Merleau-Ponty (como estética, psicoanálisis, etología o pensamiento político); diálogo filosófico desprejuiciado pero riguroso desde el punto de vista conceptual, metafísico o epistemológico, con la escuela fenomenológica y también con las ciencias del viviente humano, naturales o no naturales. Tales son las improntas que, renovadas, re-instituidas, encontramos en estos escritos. De esta forma homenajeamos los 75 productivos años de Fenomenología de la percepción tanto como a aquellos pioneros que, desde enclaves existenciales particulares, nos iniciaron en su productividad póstuma. Aquella que, como bien decía Goethe y recordaba Merleau-Ponty, es la definición misma de la obra genial. 